Bull, J.M., Gutowski, M., Dix, J.K., Henstock, T.J., Hogarth, P., Leighton, T.G. and White, P.R. (2005). Design of a 3D Chirp subbottom imaging system. Marine Geophysical Researches, 26, (2-4), 157-169. (doi:10.1007/s11001-005-3715-8).

\title{
Design of a 3D Chirp sub-bottom imaging system
}

Jonathan M. Bull ${ }^{*}$, Martin Gutowski ${ }^{*}$, Justin K. Dix ${ }^{*}$, Timothy J. Henstock ${ }^{*}$, Peter Hogarth ${ }^{\S}$, Timothy G. Leighton ${ }^{\ddagger} \&$ Paul R. White ${ }^{\ddagger}$

* School of Ocean and Earth Sciences, Southampton Oceanography Centre, European Way, Southampton, SO14 3ZH, United Kingdom; bull@soton.ac.uk

${ }^{\S}$ GeoAcoustics Ltd., Shuttleworth Close, Gapton Hall Industrial Estate, Great Yarmouth, Norfolk, NR31 ONQ, United Kingdom

${ }^{\ddagger}$ Institute of Sound and Vibration Research, Southampton University, Highfield, Southampton, SO17 1BJ, United Kingdom

Corresponding author's address:

Email: bull@soton.ac.uk

Key words: Chirp, 3D seismic, High-resolution seismics, 3D Chirp, seismic sources

Running Head: Design of a 3D Chirp System 


\begin{abstract}
Chirp sub-bottom profilers are marine acoustic devices that use a known and repeatable source signature $(1-24 \mathrm{kHz})$ to produce decimetre vertical resolution cross-sections of the subseabed. Here the design and development of the first true 3D Chirp system is described. When developing the design, critical factors that had to be considered included spatial aliasing, and precise positioning of sources and receivers. Full 3D numerical modelling of the combined source and receiver directivity was completed to determine optimal source and receiver geometries. The design incorporates 4 source transducers $(1.5-13 \mathrm{kHz})$ that can be arranged into different configurations, including Maltese Cross, a square and two separated pairs. The receive array comprises 240 hydrophones in 60 groups whose group-centres are separated by $25 \mathrm{~cm}$ in both horizontal directions, with each hydrophone group containing four individual elements and a pre-amplifier.

After careful consideration, it was concluded that the only way to determine with sufficient accuracy the source-receiver geometry, was to fix the sources and receivers within a rigid array. Positional information for the array is given by a Real Time Kinematic GPS and attitude system incorporating four antennas to give position, heading, pitch and roll. It is shown that this system offers vertical positioning accuracy with a root-mean-square (rms) error less than $2.6 \mathrm{~cm}$, while the horizontal positioning rms error was less than $2.0 \mathrm{~cm}$. The system is configured so that the chirp source signature can be chosen by software aboard the acquisition vessel.

The complete system is described and initial navigational and seismic data results are presented. These data demonstrate that the approach of using a fixed source-receiver geometry combined with RTK navigation will provide complete 3D imaging of the sub-surface.
\end{abstract}




\section{Introduction}

Developments of sub-bottom profiling systems over the last fifteen years have focused on the production of new source signatures to improve the signal-to-noise ratio of the acquired data, the resolution of the systems and the ability to acquire quantitative data on the physical properties of the sub-surface. A significant development in this area has been the production of swept frequency (Chirp) sources that improve on all three of these requirements, through a combination of having wide bandwidths, improving resolution, and controlled source signatures that facilitate both improved signal-to-noise ratio and the potential for quantitative analysis (Schock et al., 1990; 1994). These sources typically operate in a range of $1-24 \mathrm{kHz}$, offer vertical resolution on a decimetre scale in the top c. $20-30 \mathrm{~m}$ of unconsolidated sediments, and are being used increasingly for marine exploration and extraction, military applications, environmental assessments and geological and archaeological research. Work at the Southampton Oceanography Centre, over the last decade, has focused on the acquisition, processing and interpretation of data from Chirp systems deployed with Differential GPS and mounted on novel platforms to enable operation in shallow water environments (Bull et al., 2003; Bull et al. 1998; Quinn et al. 1997a and b; Quinn et al., 1998). Bull et al. (2003) demonstrated the potential for migration of high-frequency 2D Chirp data. However, 2D data never provides information on cross-dip variation and so in the migration of such data the final reflection points are constrained to lie within the plane of the section. Therefore in the presence of rapidly varying 3D topography (i.e. discrete targets) such two-dimensional migration is inevitably an imperfect process.

The ability to collect a high frequency 3D volume will ensure that the limit on the minimum dimensions of objects that can be detected will be controlled by the acoustic characteristics of the source and receiver array rather than the survey design. Furthermore, the ability to locate accurately both source and receiver groups will facilitate full threedimensional migration in which reflection points can be moved in any azimuthal direction. The ability to conduct full 3D migration has three primary effects: (1) reflections from dipping reflectors will be correctly repositioned; (2) energy scattered from small features will be focused at the scatterer location; and (3) the output volume will be evenly sampled in all three dimensions. The first two of these also act to improve the lateral resolution of the data from the scale of a Fresnel zone (order of a few metres) to the dominant wavelength of the source signal $(10-20 \mathrm{~cm})$. The limit on the lateral resolution of the data will be ultimately controlled by spatial aliasing criteria based on the horizontal apparent wavelength of the arrivals. 
There have been several systems that sought to take seismic acquisition and processing methodologies, developed by the hydrocarbon exploration industry, and apply them at higher frequencies to obtain sub-metre horizontal and vertical resolution. A major issue and factor controlling the relative success of these systems has been the absolute and relative positioning of source and receivers. Versteeg et al. (1992) describe a system using boomer and watergun sources with mid-frequencies of c. $1 \mathrm{kHz}$ on a catamaran towing 12 dual-channel streamers. The catamaran was positioned using a land-based laser-ranging theodolite system with a single corresponding prism crown on the catamaran, but there was no control on the position of the hydrophones. Marsset et al. (1998) discussed processing techniques applicable to this system and showed improvement in data quality using migration algorithms.

Missiaen et al. (2002) and Missiaen (2004, this volume) describe a compact flexible 3D system comprising a RIB, flanked by six modular floating frames with an overall width of $14 \mathrm{~m}$ from which 8 dual-channel streamers are deployed. A boomer source with a central frequency of $2 \mathrm{kHz}$, together with Real Time Kinematic (RTK) GPS positioning, is used to locate accurately the source. Diviacco and Wardell (2004, this volume) describe processing procedures that use seafloor reflection time information to correct for small-scale variations in the relative positions of source and receiver. This is necessary because the receiver positions are not explicitly known.

Schock et al. (2001) describe a buried object scanning sonar that uses a chirp source. This system operates with a line source array that uses beam-steering and a planar receiving array with 32 hydrophones distributed in an area of less then $1 \mathrm{~m}^{2}$. This system is shown to be capable of collecting a narrow 3D volume along the track of the vehicle, but the authors do not address absolute positioning or create a $3 \mathrm{D}$ volume using information from adjacent lines.

High frequency (here defined as above $1 \mathrm{kHz}$ central frequency) seismic systems that can produce true 3D data volumes have been hampered by lack of absolute positional control on the positions of sources and receivers. In this paper the development of a true 3D Chirp sub-bottom profiling system is described. Through a combination of RTK GPS positioning and attitude systems, and a fixed source-receiver geometry, a solution to the problems of positional control is demonstrated.

The approach that is taken here is to review the underlying physical fundamentals required to produce a fully sampled accurate 3D volume. A model of energy scattered from the seabed, incorporating directivities of all the system components, is developed and used to assess spatial 
aliasing. Static and dynamic tests on the positioning solution used in the 3D array are described. The final design of the 3D Chirp sub-bottom imaging system is illustrated and explained, before initial results from data collected in August 2003 are presented.

\section{Physical Fundamentals}

Lower frequency $(10-200 \mathrm{~Hz})$ 3D seismic volumes have been collected for the oil industry for several decades. Although many of the principles and seismic processing algorithms are directly applicable to high frequency data, there needs to be careful consideration of the physical basis for the collection of true 3D data. The following need to be carefully considered: (1) Horizontal positioning and spatial aliasing; (2) Horizontal and vertical positioning accuracy requirements; and (3) Source signature considerations and frequency aliasing. The first two of these are discussed in detail in the following sections. Gutowski et al. (2002) presented the results of the design and testing of different chirp source signatures and discussed methodologies for assessing the relative merits of different sweeps with regard to their penetration and resolution characteristics. This work identified that an optimal chirp sweep for both penetration and resolution used a broad bandwidth and a sinesquared taper function (Figure 1). With regard to frequency aliasing, the sampling interval (31.25 $\mu \mathrm{s})$ of the digital acquisition system used in this work was sufficient to ensure that the highest frequencies contained within the source sweeps $(13 \mathrm{kHz})$ were over-sampled.

\section{Horizontal resolution and spatial aliasing}

The horizontal resolution of seismic data indicates how closely spaced two point diffractors may be while still being distinguished. In raw data this is controlled by the Fresnel zone radius, the region over which energy scattered at depth interferes constructively at the surface. Theoretically the horizontal resolution may be improved by processing algorithms such as migration to be as low as $\lambda / 2$, where $\lambda$ is the dominant wavelength, although in practice it will be limited by the range of horizontal wave numbers present in the data.

In order to apply wavefield-based methods such as migration, it is essential that the recorded energy is fully sampled in space as well as in time, i.e. that spatial aliasing of the data is avoided (e.g., Yilmaz, 1987). With a receiver spacing $\Delta x_{\text {rec }}$ in the array, and energy with wavelength $\lambda$ incident on the array at angle $\alpha$ from the vertical, this requires that

$$
\Delta x_{\text {rec }}<\frac{\lambda}{2 \sin \alpha} .
$$


The receiver spacing required for this is smaller for shorter wavelengths or for incident energy that travels more horizontally. Clearly there is an additional factor that for a fixed number of channels, the smaller the receiver spacing used, the smaller will be the overall footprint of the array, and the more closely spaced must be individual sail-lines. Hence for efficiency $\Delta x_{\text {rec }}$ must be maximized while minimizing potential problems of spatial aliasing. The most likely origin for problematic aliased energy is by scattering at the seabed because, for a given recording time, the energy will travel most shallowly and have the highest amplitudes. Hence the effect of such scattered energy, including the directivities of each element in the hardware, has been modelled.

\section{Modelling of energy scattered from the seabed}

The model calculates the amplitude of the energy that would be returned from a given point on the seafloor for specular reflection at that point with a reflection coefficient of 1 and assuming no transmission losses in the water column; this will overestimate the amplitude of any possible return. In this analysis we are modeling the worst-case scenario for spatial aliasing. It is assumed that the source array and hydrophone group are centered at the same location; the actual offset between these is less than about $1 \mathrm{~m}$ (which is small compared with typical likely water depths of $10-30 \mathrm{~m}$ ). The recorded amplitude of the frequency dependent energy returned from any given point on the seabed will be the product of the following factors: the directivities of the individual source transducer, the transducer array, the hydrophone group; and geometric spreading (Figure 2). All the directivities have been normalized by the amplitude of the specular reflection from the seabed immediately beneath the origin. The water depth and the recording time control the region over which the amplitudes are calculated; the example in Figure 3 assumes a frequency of $7.25 \mathrm{kHz}$ and a water depth of $20 \mathrm{~m}$.

The single transducer directivity due to the finite size of the transducers and any intrinsic effects, $D_{t}$, was provided by Neptune Sonar Ltd for the T135 transducers used in the system (Figure 2a). Although the discussion here focuses on a transducer configuration in which transducers were arranged as a Maltese Cross (Figure 2b), a number of other different transducer configurations were modelled including four arranged as a square, and two as a pair. The directivity of the Maltese Cross arrangement for four point transducers, $D_{a}$, was calculated based on the intended source array geometry (Figure 2b); the wide axis of the Maltese cross is in the y direction leading to a reduced footprint. The hydrophone group consists of four elements at $6.25 \mathrm{~cm}$ spacing, leading to a directivity $D_{r}$; the long axis of the element is oriented parallel to the x direction, so that there is no array effect in $\mathrm{y}$ (Figure 2c). Each individual hydrophone element is much smaller than the 
wavelengths used so that they may be considered omnidirectional. The effect of geometrical spreading is considered to have spherical symmetry and is simply $1 / r^{2}$.

The final amplitude, $A$, as a function of seabed location can be calculated as

$$
A=\frac{D_{t} \cdot D_{a} \cdot D_{r}}{r^{2}}
$$

and is shown in Figure 3a. Note that the seabed returns with significant amplitude are limited to a zone close to the specular direction; although the transducer array response has strong sidelobes, these are suppressed by the directivity of the individual transducers and the spreading factor. The perpendicular orientation of the long axes of the source array and hydrophone group also minimises the overall footprint.

Figure $3 \mathrm{~b}$ shows cross-sections through the returned amplitude function along the $\mathrm{x}$ and $\mathrm{y}$ axes. For each point on the cross-section the apparent wavelength, $\lambda$ app $=\lambda / \sin \alpha$ is also calculated. This modelling enabled the selection of an appropriate receiver spacing that would avoid spatial aliasing, but still provide a practicable solution that will enable the acquisition of a true 3D volume. Figure $3 \mathrm{~b}$ shows that for aliased energy to have amplitudes less than $10 \%$ of that reflected immediately beneath the array if it is scattered in the crossline direction, and less than $25 \%$ in the inline direction, then apparent wavelengths of greater than $0.5 \mathrm{~m}$ must be fully sampled. This shows that a receiver group spacing of $0.25 \mathrm{~m}$ would be necessary, although this is an extreme case as we overestimate the amplitude of any recorded return. In summary, for the range of frequencies generated by the chirp source signature, it is concluded that a receiver separation of $25 \mathrm{~cm}$ in both $\mathrm{x}$ and y directions will result in negligible spatial aliasing. 


\section{Horizontal and Vertical Positioning Accuracy Requirements \\ Requirements}

The accuracy required for the positioning of the source and receiver elements of the 3D chirp system depends on the characteristics of the source signature used. In order to ensure that requirements are met for all sweeps, the source signature which produces the tightest autocorrelation function (Klauder wavelet) to be used with the system is considered. This signature (Figure 1) has a linear Instantaneous Frequency Function on a frequency band from $1.5-13 \mathrm{kHz}$ and a sine-squared taper function with a mean frequency of $7.25 \mathrm{kHz}$ (for a full discussion of Chirp source signatures see Gutowski et al., in 2002).

The required accuracy in the vertical direction results from the necessity to stack seismic traces in a common mid-point bin defined in the geometry assignment during the 3D data processing. In order to avoid destructive interference by stacking the seismic traces, the error in its associated vertical positions need to be smaller then the distance between the main-lobe peak and the side-lobe trough of the signature's Klauder wavelet. This distance is $60 \mu$ s for the Klauder wavelet considered (Figure 1), equivalent to $9 \mathrm{~cm}$ assuming a p-wave velocity of $1500 \mathrm{~m} / \mathrm{s}$ in water. In order to reconstruct the wavefield adequately, the horizontal position needs to be known within a quarter of the wavelength, $\lambda / 4$. For the Klauder wavelet discussed, and the assumptions made, this is $5 \mathrm{~cm}$. Furthermore, the positions need to be measured reliably with an update rate similar to the seismic shot rate. In summary navigational control is needed which will provide less than $9 \mathrm{~cm}$ vertical and less than $5 \mathrm{~cm}$ horizontal resolution.

\section{Positioning solution}

Positioning of the 3D chirp system is greatly simplified if the source and receiver elements are mounted on a plane and rigid array, so that the source-receiver offsets are known a priori. Given the absolute positioning accuracy required in both horizontal and vertical planes, the 3D chirp system uses a Real Time Kinematic GPS System (the Sagitta02 Long Range Kinematic, LRK $\mathrm{TM}_{\text {, }}$ GPS system) and a GPS attitude determination system (ADU5) both supplied by Thales Navigation. The knowledge of absolute position on the array together with its orientation: heading, pitch and roll allows the calculation of the absolute position of the source and receiver element positions. The RTK system uses a reference station with a GPS antenna at a known geographic location, sending GPS correction information via an UHF radio link to the roving station, providing the highly accurate absolute position of the roving station's GPS antenna in real-time. The GPS attitude determination is based on differential carrier phase measurements between the four antennas. One antenna is shared by the two systems. 
In order to test the suitability of the system for marine work, two experiments were carried out. Firstly, to test system accuracy performance, the attitude and position were recorded in a static position over a period of time. Secondly, the system was mounted onto a catamaran and towed behind a survey vessel to simulate its application. Although the tests were completed using a catamaran rather than the final 3D chirp system, the worst-case errors in receiver element position could be simulated in a straightforward manner.

\section{Static test}

The GPS antennas were mounted onto a catamaran as shown in Figure 4. Antenna 1 is shared by the RTK GPS and the GPS attitude system; antennas 2, 3 and 4 are used only by the GPS attitude system. The radio-link with a local reference station was established and data recorded over a period of c. 20 minutes.

Figure 5a shows the absolute position data for Antenna 1 with the deviation of the $\mathrm{x}-, \mathrm{y}-$, and $\mathrm{z}$-coordinate values from their mean value. Data from the attitude system are shown in Figure $5 \mathrm{~b}$ showing the deviation of heading, pitch and roll for the catamaran from their mean value. Figure 5c shows the coordinates for a simulated element position, that is offset by $2 \mathrm{~m}$ in longitudinal and 2.5 $\mathrm{m}$ in transversal direction from antenna 1, which corresponds to the greatest offset between the GPS antenna and a receiver element on the final 3D chirp array (see later). Figure 5d shows an $\mathrm{x}-\mathrm{y}$-plot of this elements positional variation with time. The accuracy of the attitude system can be expressed as root-mean-square (rms) error, which, for the static test was $0.16^{\circ}$ for heading, $0.32^{\circ}$ for pitch and $0.22^{\circ}$ for roll.

The error in the absolute position of Antenna 1 is summarised in Table I, together with the simulated error in the position of the element furthest from the Antenna 1 in the final 3D array. The element position error includes the error in the absolute position of Antenna 1 and the errors in heading, pitch and roll. The element position error represents the worst-case expected error in position for a hydrophone element on the final 3D array. Hence positioning errors in the final 3D array are expected to be less than or equal to $\pm 1.7 \mathrm{~cm}$ in the horizontal direction, and $\pm 2.5 \mathrm{~cm}$ in the vertical direction. These errors are much less than the positioning requirement for the $3 \mathrm{D}$ array ( $5 \mathrm{~cm}$ in the horizontal and $9 \mathrm{~cm}$ vertically) discussed previously.

\section{Dynamic test}

Although the static tests were successful, a dynamic experiment was completed to check that the positioning system could rapidly respond to changes in the array position due to currents and wave action. The catamaran was towed behind a small survey vessel in Southampton Water (UK) in a calm sea state. In order to achieve roll and pitch movements the catamaran was navigated through 
the wash of passing ships. Figure 6 shows an example in which the wavefront of the wash travels in direction c. $220^{\circ}$. Figure $6 \mathrm{~b}$ shows the trajectory of the catamaran describing a bend. Figure 6a shows that the heading changes from $200^{\circ}$ to $130^{\circ}$ at point $\mathrm{A}$, where the catamaran is parallel to the incoming wavefront. It experiences a roll motion with an amplitude of up to $12^{\circ}$ and change in the vertical position of up to c. $40 \mathrm{~cm}$. It turns further while the roll motion decreases and the pitch motion increases. At point B the heading value is c. $55^{\circ}$ it is nearly perpendicular to the wavefront, and it experiences a maximal pitch motion of c. $5^{\circ}$, reflected in a change of vertical position of up to $32 \mathrm{~cm}$. The towing behaviour of the catamaran is such that it is reluctant to point directly into the wash, and is towed with a yaw angle as apparent from the difference between the measured heading and the heading values derived from the trajectory. The experiment lasted for c. one hour, during which time the vertical position value linearly decreased by c. $1 \mathrm{~m}$, corresponding to the tidal changes in the area. Throughout the experiment the system reliably provided dynamic position and attitude information.

The static experiment showed that measured rms errors are within the calculated accuracy requirements for a 3D Chirp seismic system. The dynamic experiment simulated the positioning of the seismic system during a survey, and showed that the system could reliably record changes in the position of the catamaran due to wave and current action.

\section{D Chirp Design}

In the preceding section it has been demonstrated that: (1) for the highest resolution sweep a sampling interval of $31.25 \mu$ s will result in over-sampled data; (2) that a receiver spacing of $25 \mathrm{~cm}$ in $\mathrm{x}$ and $\mathrm{y}$ directions will avoid significant spatial aliasing of the seismic data; (3) that a RTK GPS system together with an attitude system would give the required horizontal and vertical accuracy in positioning for the system. In this section the final design for the 3-D Chirp profiling system is described and illustrated.

As mentioned already one of the major problems in acquiring 3D data volumes at high frequencies is uncertainty in the position of sources with respect to receivers. The solution reported here is to use a rigid array with known source and receiver relative positions. Figure 7 shows technical drawing of the 3D-Chirp system. The four transducers are shown arranged in the preferred Maltese Cross configuration, which gives enhanced directivity along-track, within the centre of the array together with the electronics bottles that drive the transducers. Careful consideration had to be given to achieving neutral buoyancy. This was solved by using a composite, low-density, reinforced foam central panel to support the transducers and electronics bottles. The design is modular so that 
the four transducers can be rearranged in a standard square configuration in the centre of the array, or as two pairs towards the edge of the mat to maximise ground coverage.

Eleven longitudinal 2.25 m-long composite tubes containing the hydrophone groups are fitted on the underside (Figure 7b). Each longitudinal sections contains between 4 and 6 hydrophone groups, such that there are 60 independent hydrophone groups within the array. Hydrophones positions can be changed within the array to accommodate different transducer positions. Each hydrophone group is $25 \mathrm{~cm}$ long and contains a pre-amplifier and four elements, each separated by $6.25 \mathrm{~cm}$, which sum the arriving energy, and facilitate an increase in signal-to-noise. The design ensures that the hydrophone groups are protected from reverberation off the water-surface. The array has been designed so that hydrophone cabling is accommodated within the tubes, before being collected together within two splice blocks at the front of the array. The data is then sent up two cables to the recording system aboard the acquisition vessel.

The four GPS antennas at the corners of the array provide positioning. Each of the antennas is $1 \mathrm{~m}$ above the top of the array, and as described previously one of these antennas provides absolute position, while all antennas are used in the attitude system to give heading, pitch and roll. Data from all four antennas is then used to produce the absolute position of each source point and hydrophone group within the array to centimetric accuracy. This level of navigational accuracy enables not only correct within-line location of targets but also the effective merging of data from adjacent lines, including corrections for tidal variability.

The array has been hydrodynamically designed to tow smoothly and to ride swell and wave activity without tilting and to avoid acoustic noise generation. The total weight of the array including all elements is $176 \mathrm{~kg}$ and it has been designed to be deployable from small vessels.

The acoustic data from the system is recorded using a 60-channel Geometrics Strataview R60 system and is merged with the centimetric accuracy navigation data for later data processing. Seismic processing is completed in three phases: geometry processing, including binning; 3D migration; and then standard Chirp processing techniques as described by Quinn et al. (1998). Finally the data is taken into a 3D seismic interpretation workstation for interpretation and target recognition. 


\section{Testing and Initial Results from the 3D Chirp System}

The array was constructed in July 2003, and tested within the Solent (U.K.) during August 2003 over a two-week period. The system proved to be neutrally buoyant and towed in a stable manner (Figure 8). The GPS antennas sit c. $0.7 \mathrm{~m}$ above the surface of the water with the base of the transducers and hydrophones sitting c. $0.4 \mathrm{~m}$ beneath the surface of the water. During the trial the system was tested in sea-states up to a Force 4 and an optimal towing speed through the water was found to be $2.0 \mathrm{~ms}^{-1}$. The sine-squared source signature shown in Figure 1 was used throughout the trials with the source transducers arranged as a Maltese Cross.

Seismic data was acquired within the west Solent (Figure 9) over an area known to contain dipping and faulted limestones and mudstones (Daley et al., 1979). An area $80 \mathrm{~m}$ wide by $600 \mathrm{~m}$ long was surveyed using repeated lines. To ensure that the area was completely sampled, sufficient lines were collected so that the area was covered with $1 \mathrm{~m}$ spaced lines.

The RTK GPS absolute positional data and attitude data was used to provide the position of the centre of the source and the 60-receiver elements. The positioning systems worked reliably throughout the trial and, in this paper, one example of the precise positional control afforded by the navigation system employed on the 3D array is shown. Figure 9 shows receiver positions data for two survey lines, with each dot representing a receiver. The scale shows the range in variation in vertical height of the array during the collection of the two profiles. This figure illustrates the longer-term change due to tidal variation and shorter-term changes due to movements of the array. Note the tilting of the array during a line turn so that one corner of the array was up to $50 \mathrm{~cm}$ higher than the diagonally opposite corner.

The midpoints of the two lines illustrated in Figure 9 have been binned into a Common Depth Point (CDP) grid with $12.5 \mathrm{~cm}$ spacing and static corrections have been applied using the vertical position of the source and receiver, assuming a p-wave velocity in water of 1500 $\mathrm{m} / \mathrm{s}$. The inset box shows three traces within a CDP bin with the prominent arrival being the seafloor reflection. The two left traces represent channel 9 and 15 from shot 224, the third trace represents channel 51 from shot 1660 collected 20 minutes later during the survey. The source and receiver heights changed c. $14 \mathrm{~cm}$ in between the shots due to the tides. The seafloor reflections line up within $20 \mu$ s two-way time, corresponding to a vertical height of $1.5 \mathrm{~cm}$, proving that the position information that is used to correct for the static shifts is within the errors calculated earlier. Analysis of the static corrections in other multi-fold CDP 
bins shows similar results.

Data is currently (November 2003) being processed and prepared into a 3D volume. In this paper a single representative $2 \mathrm{D}$ line showing the quality of the data is shown. In this profile the data from 5 in-line hydrophones has been stacked, correlated, band-pass filtered (01.5-13-15 kHz) and had Instantaneous Amplitude applied. As can be seen in Figure 10 the Chirp data images dipping Limestone and Mudstone bands down to c. $25 \mathrm{~m}$ beneath the seabed where the seabed multiple occurs. Data was reliably recorded on all seismic channels.

\section{Conclusions}

This paper outlines the construction of a 3D chirp system for the imaging at decimetre resolution (in $\mathrm{x}, \mathrm{y}$ and $\mathrm{z}$ ) the subsurface to a depth of c. $20 \mathrm{~m}$. The paper discusses the initial concept, numerical modelling of key acoustic, positional and engineering parameters, and finally the production and testing of the built array. The design aimed to provide, for a $1.5-13 \mathrm{kHz}$ Chirp source, a CDP spacing of $12.5 \mathrm{~cm}$ while ensuring that spatial and frequency aliasing was minimised. This was achieved through the construction of a rigid geometry, to house 60 purpose built hydrophone groups, that was keyed in to an RTK-GPS position and attitude system, giving $\mathrm{x}, \mathrm{y}, \mathrm{z}$ data within pre-determined limits. The system has been tried over an area of complex faulted geology, and the preliminary results show that 60 channels of high-quality Chirp data has been collected in tandem with the co-registered RTKGPS navigation data. Preliminary processing shows that $12.5 \mathrm{~cm}$ CDP bins, containing data from more than one survey line, can be produced within the required accuracy in position. Work is continuing on the production of the final data volume.

Future work will include the development of a new acquisition system to allow shotrates of at least four times per second and increasing the size of the array. This last change will allow greater sail-line intervals, and more cost-effective surveying.

\section{Acknowledgements}

The authors acknowledge support from the Engineering and Physical Sciences Research Council/Joint Grant Scheme (GR/R 12695/01), GeoAcoustics Ltd, and the English Heritage ALSF fund for this work. The authors thank Dave Whatley, Mike Street, Adrian Lester, and James Chitty for their detailed help in developing the design. Ray Collins and John Davis are thanked for their practical assistance. 


\section{References}

Bull, J.M., Minshull, T.A., Mitchell, N.C., Dix, A.I., Thors, K., and Best, A.I., 2003, Fault and magmatic interaction within Iceland's western rift over the last 9 kyr. Geophys. J. Int. 154, F1-F8.

Bull, J. M., Quinn, R. and Dix, J. K., 1998, Reflection Coefficient Calculation from Marine High Resolution Seismic Reflection (Chirp) Data and Application to an Archaeological Case Study. Mar. Geophys. Res. 20, $1-11$.

Daley, B., Edwards, N. and Insole, A. N., 1979, Lithostratigraphical nomenclature of the English Paleogene Successions. Geological Magazine 116, 65-66.

Diviacco, P., and Wardell, N., 2004, Pre-processing of 3D VHR seismic data. Mar. Geophys. Res., this volume

Gutowski, M., Bull, J.M., Henstock, T.J., Dix, J.K., Hogarth, P., Leighton, T.G., White, P.R., 2002, Chirp subbottom profiler source signature design and field testing. Mar. Geophys. Res. 23: 481-492.

Marsset, B., Missiaen, T., Noble, M., Versteeg W., Henriet J.-P., 1998, Very high resolution 3D marine seismic data processing for geotechnical application. Geophysical Prospecting, 46, 105-120.

Missiaen, T., 2004, VHR 3D marine seismics for shallow water investigations: some practical guidelines. Mar. Geophys. Res., this volume

Missiaen, T., Versteeg, W., Henriet J.-P., 2002, A new 3D seismic acquisition system

for very high resolution and ultra high resolution shallow water studies 2002, First Break 20, 227-232

Quinn, R., Bull, J.M. and Dix, J. K., 1997(a), Buried scour marks as indicators of paleo-current direction. Mar. Geol. 140, 405-413.

Quinn, R., Bull, J.M. and Dix, J. K., 1997(b), Imaging Wooden Artefacts using Chirp Sources. Archaeol. Prospect. 4, 25-35.

Quinn, R., Bull, J.M. and Dix, J. K., 1998, Optimal processing of marine high-resolution seismic reflection (Chirp) data. Mar. Geophys. Res. 20, 13-20.

Schock, S. G and LeBlanc, L. R., 1990, Chirp Sonar: New Technology For Sub-Bottom Profiling. Sea Technology 31(9), 35-43.

Schock, S. G., LeBlanc, L. R. and Panda, S., 1994, Spatial and Temporal Pulse Design Considerations for a Marine Sediment Classification Sonar. IEEE J. Ocean. Eng._19(3), 406-415.

Schock, S.G., Tellier, A., Wuld, J., Sara, J., and Ericksen, M., 2001, Buried object scanning sonar. IEEE J. Ocean. Eng. 26, 677-689.

Verstegg, W., Verschuren, M., Henriet, J-P., and De Batist, M., 1992, High resolution 3D and pseudo-3D seismic investigations in shallow water environments. European conference on underwater acoustics. London. Elsevier Applied Science. 497-500.

Yilmaz, O., 1987, Seismic Data Processing. Society of Exploration Geophysicists, Tulsa, OK. 


\section{Tables}

\begin{tabular}{ccc}
\hline & $\begin{array}{c}\text { LRK }^{\text {TM }} \text {-GPS position } \\
\text { antenna 1 rms }[\mathrm{mm}]\end{array}$ & $\begin{array}{c}\text { LRK }^{\text {TM }} \text {-GPS position } \\
\text { receiver element rms [mm] }\end{array}$ \\
\hline x & 7.9 & 10.1 \\
y & 13.8 & 17.0 \\
z & 20.3 & 25.3 \\
\hline
\end{tabular}

Table I. Positioning accuracy expressed as root-mean-square (rms) error. Left hand column is for Antenna position 1. The right hand column is for an element position offset from Antenna 1 by $2 \mathrm{~m}$ in longitudinal and $2.5 \mathrm{~m}$ in transversal direction to simulate the worst-case error for a receiver element in the 3D chirp system.

\section{Figures}

Figure 1. Example of a chirp sweep that can be generated by the transducers on the 3D chirp system. This is a linear sweep with sine-squared taper function covering $1 / 8^{\text {th }}$ of the time duration. This sweep has a temporally short Klauder wavelet and high resolution capabilities as discussed by Gutowski et al. (2002). a) Time domain representation. b) Power spectrum with a mean frequency of $7.25 \mathrm{kHz}$. c) Spectrogram showing the linear Instantaneous Frequency Function. The greyscale represents the same $\mathrm{dB}$ scale as in the spectrum. d) Klauder wavelet, the normalised auto-correlation function of the sweep.

Figure 2. The modelled directivities of the component parts of the 3D Chirp system with contours of normalised amplitude. (a) The single transducer directivity (b) The directivity of the Maltese Cross arrangement for four point transducers; inset shows orientation of transducers (c) Hydrophone group directivity for a group comprising four elements at $7 \mathrm{~cm}$ spacing; inset shows orientation of hydrophone group. (d) The effect of geometrical spreading has spherical symmetry and is calculated as $1 / r^{2}$.

Figure 3. (a) The modelled final amplitude, $A$, as a function of seabed location with contours of normalised amplitude. (b) Cross-sections through the returned amplitude function along 
the $\mathrm{x}$ and $\mathrm{y}$ axes. For each point on the cross-section the apparent wavelength is also calculated. See main text for more discussion.

Figure 4. Experimental set-up. a) The GPS antenna positions on the catamaran as used for both the static and dynamic experiments. Antenna 1 determines the RTK GPS Position for the Sagitta02 system and is shared with the ADU5 system which additionally uses antennas 2, 3 and 4 to determine heading, pitch and roll. b) The catamaran being towed during the dynamic trials, which simulates the positioning requirements of the final 3D chirp system.

Figure 5. Static positioning experiment results using the Sagitta02 RTK (LRK $\mathrm{LM}_{\mathrm{TM}}$ ) GPS positioning and the ADU5 GPS attitude systems using configuration shown in Figure 4. a) The variation of the $\mathrm{x}, \mathrm{y}$ and $\mathrm{z}$-coordinates of Antenna 1 position from their mean value during a c 20 minutes period in which the catamaran remained static. b) The heading, pitch and roll variations for the catamaran from their mean value. c) The simulated variation of an element offset by $2 \mathrm{~m}$ in longitudinal and $2.5 \mathrm{~m}$ in transversal direction from the GPS antenna on a rigid plane determined from the RTK GPS position and attitude. This simulates the worst-case error in position of a receiver element on the $3 \mathrm{D}$ chirp system. d) The variation with time in the simulated elements position from its mean $\mathrm{x}$ and $\mathrm{y}$ position.

Figure 6. Dynamic positioning experiment using the catamaran shown in Figure 4. a) Variation in heading, z-coordinate of the RTK GPS position, roll and pitch. b) The x- and ycoordinate of the RTK GPS position.

Figure 7. Perspective view of (a) the top of the 3D Chirp system showing main elements of the design; (b) the bottom of the 3D Chirp system. Note the position of the hydrophone groups in slots on the underside of the longitudinal sections.

Figure 8. The 3D Chirp array being towed in the Solent, U.K., in August 2003. The four antennas allow the position of the source and each hydrophone group to be accurately known. The array is c. $2.7 \mathrm{~m}$ across by $2.3 \mathrm{~m}$ long. 
Figure 9. Top left inset shows the location of the study area in the Solent, U.K. The main box shows the position of two profiles $1 \mathrm{~m}$ apart with the colour coding indicating the vertical height of the array. The positions of each of the receiver elements positions are shown once per second for clarity. Note how the effects of tidal variation are evident along the lines. The position of the seismic data shown in Figure 10 is indicated along the line track. Bottom right inset shows a zoomed portion of the navigation data showing how the array tilts slightly in line turns, with one corner of the array up to $50 \mathrm{~cm}$ above the opposite corner. The inset box showing three seismic traces representing data within a single bin achieved by taking the mid-points of the two illustrated seismic profiles and binning into a Common Depth Point (CDP) grid with $12.5 \mathrm{~cm}$ spacing. The three traces come from channels on both seismic profiles and image the seabed reflection. The alignment of the seafloor reflection within the bin shows that the navigational data is known with the required accuracy for 3D surveying.

Figure 10. Example profile (position shown in Figure 9) showing rotated and faulted limestones and mudstone layers of Eocene-Oligocene age. The depth of penetration is limited to c. $25 \mathrm{~m}$ by the presence of the seabed multiple. 
a)

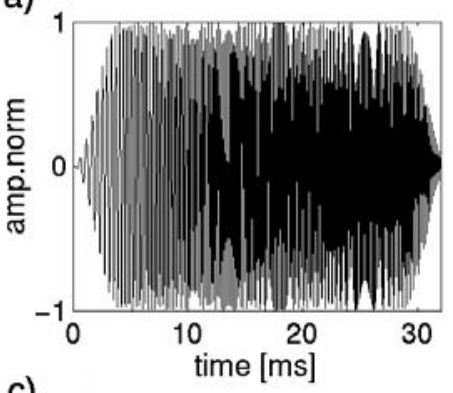

c)

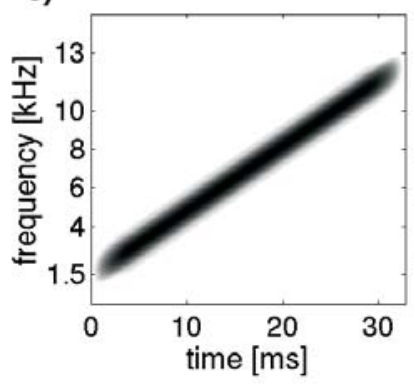

b)

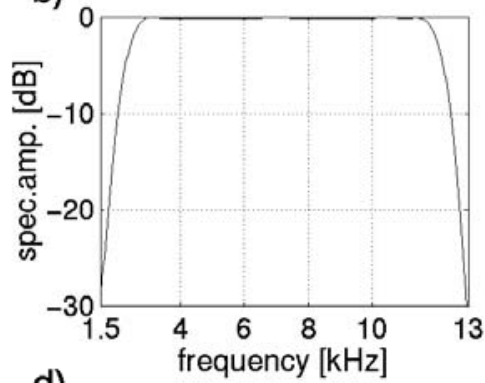

d)
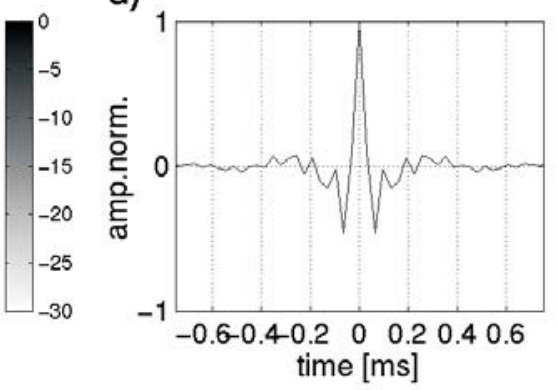

Bull et al. Figure 1 
a)

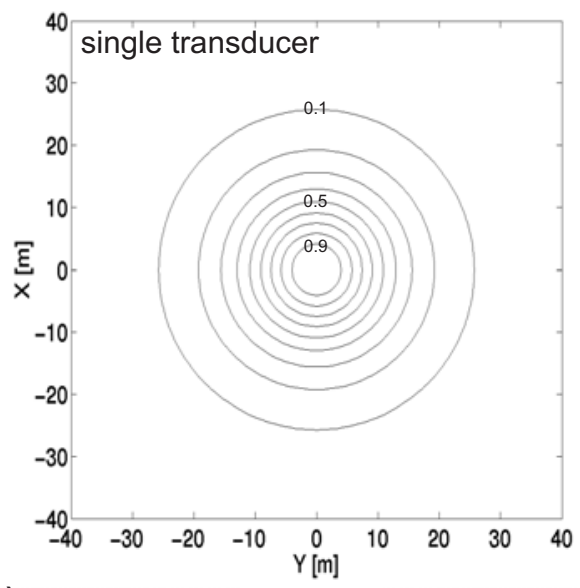

c)

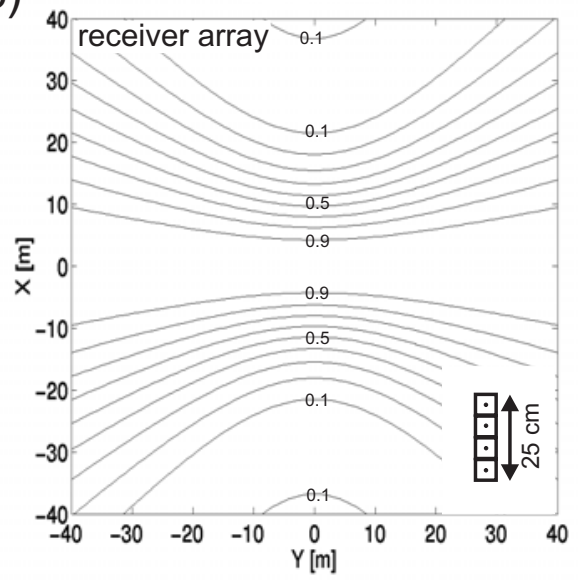

b)

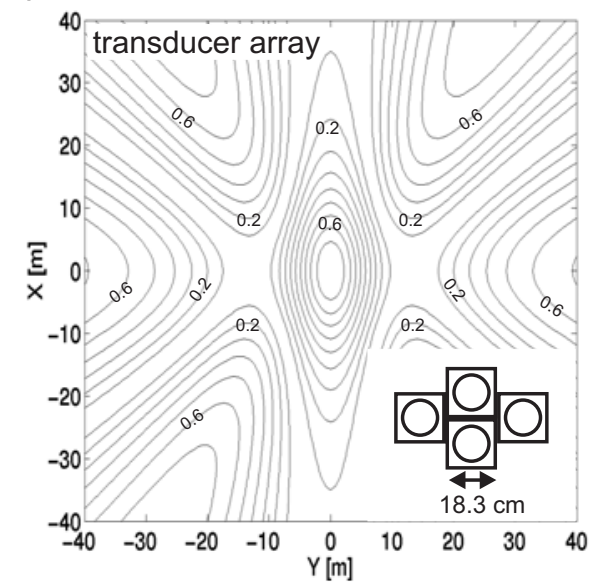

d)

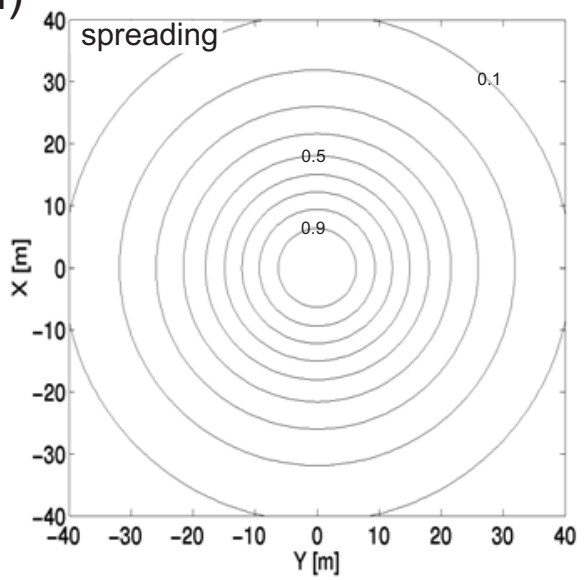

Bull et al. Figure 2 
a)

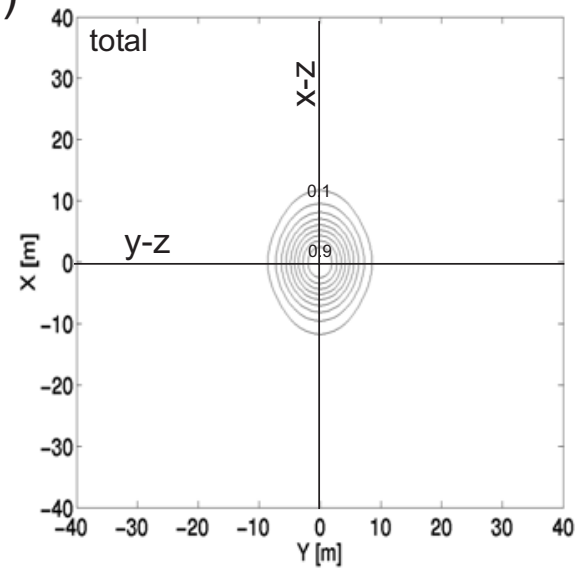

b)
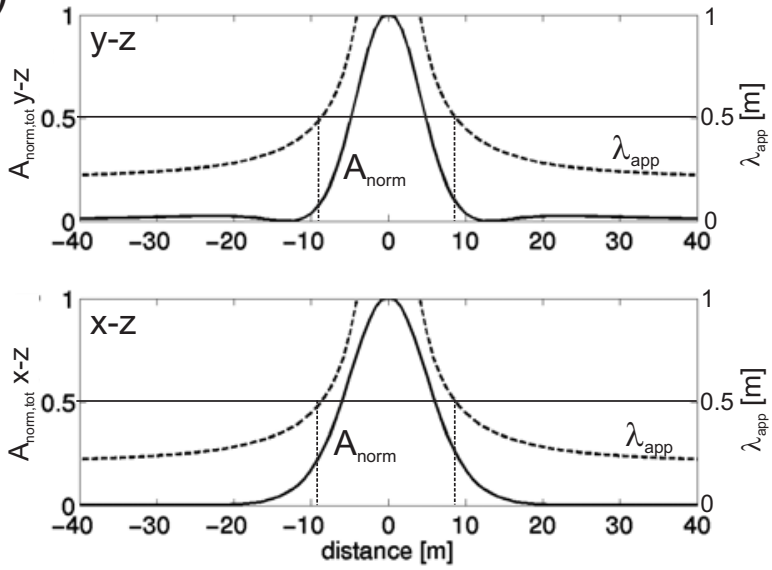

\section{Bull et al. Figure 3}




\section{GPS-antennas}

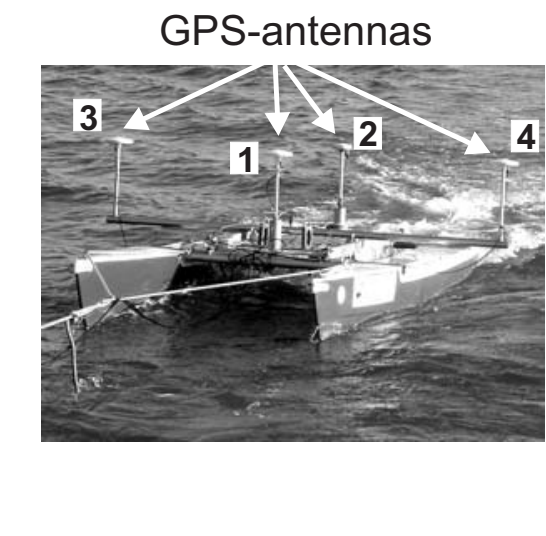

b)

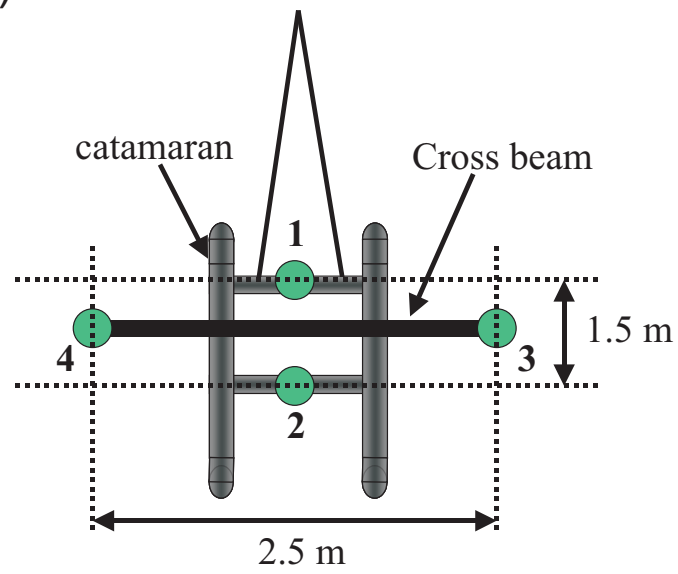

Bull et al. Figure 4

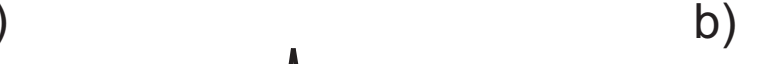

$2.5 \mathrm{~m}$

.


a)
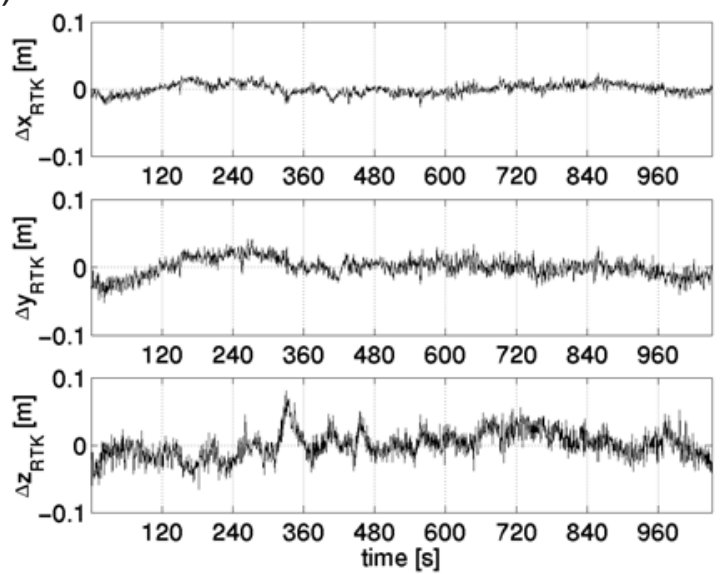

c)

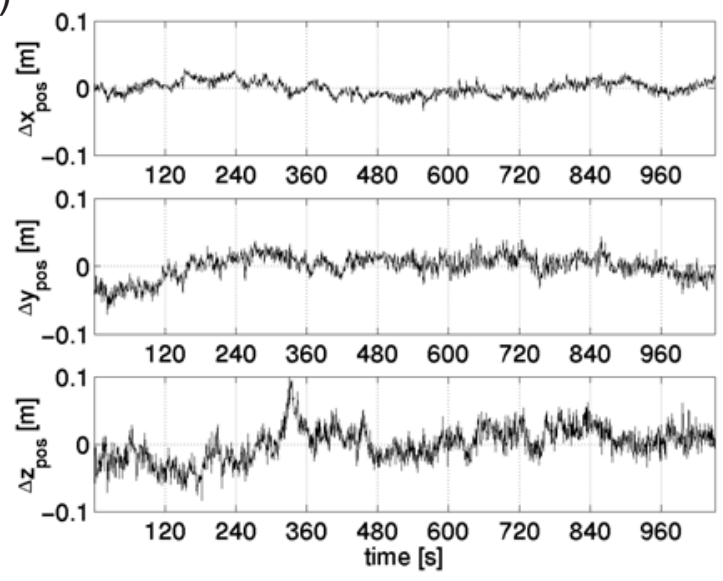

b)
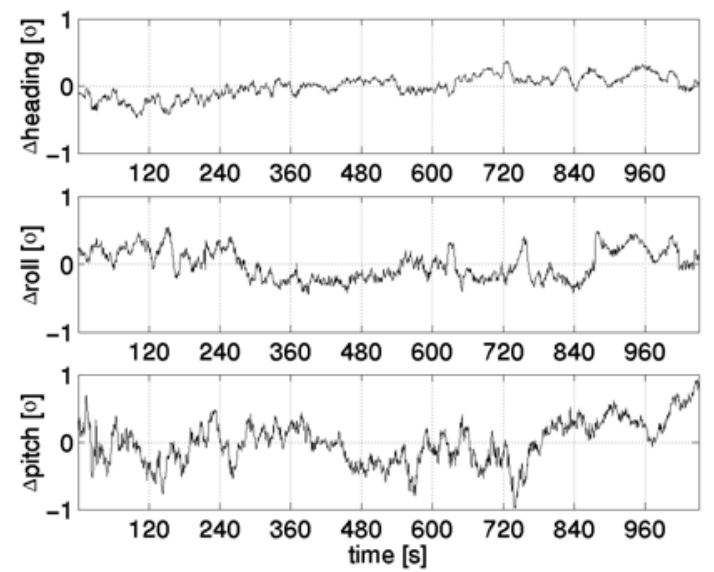

d)

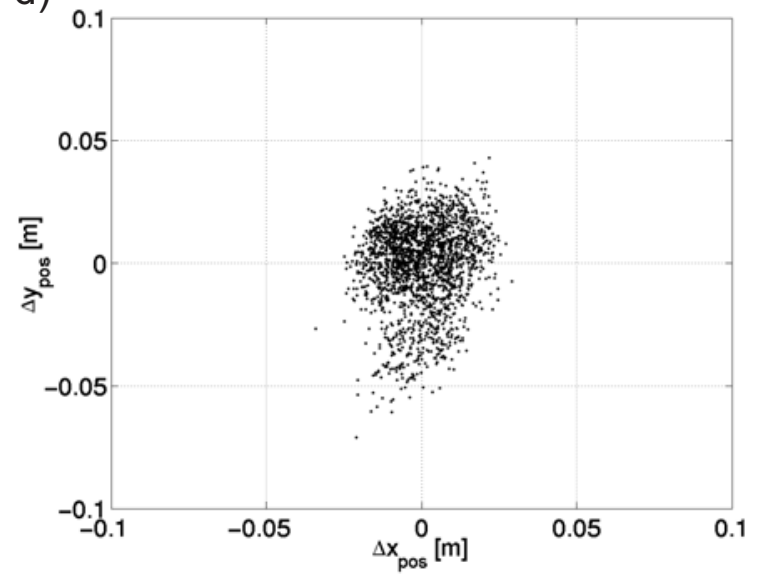




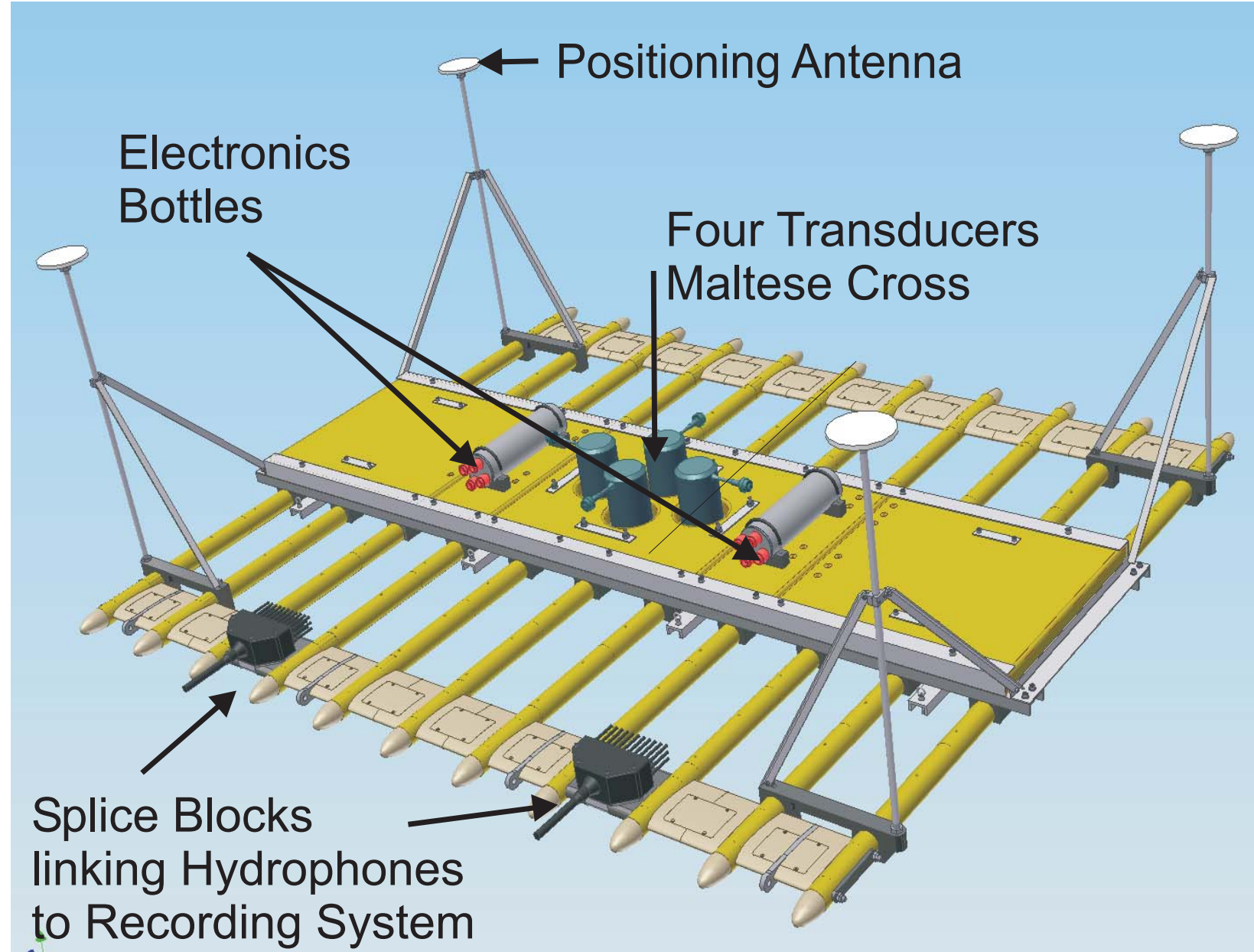

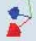

(B)

Slots for Hydrophone Groups

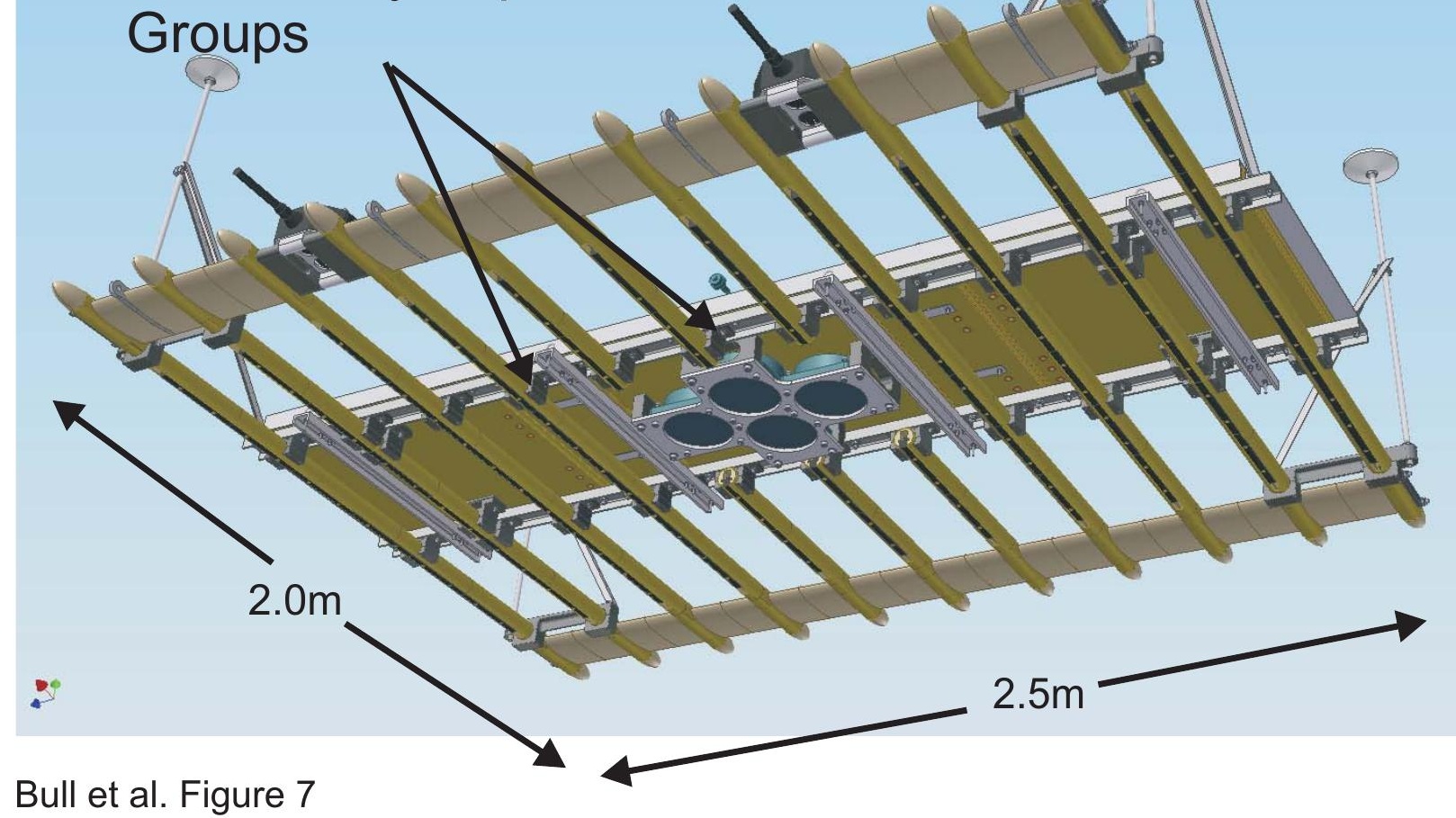




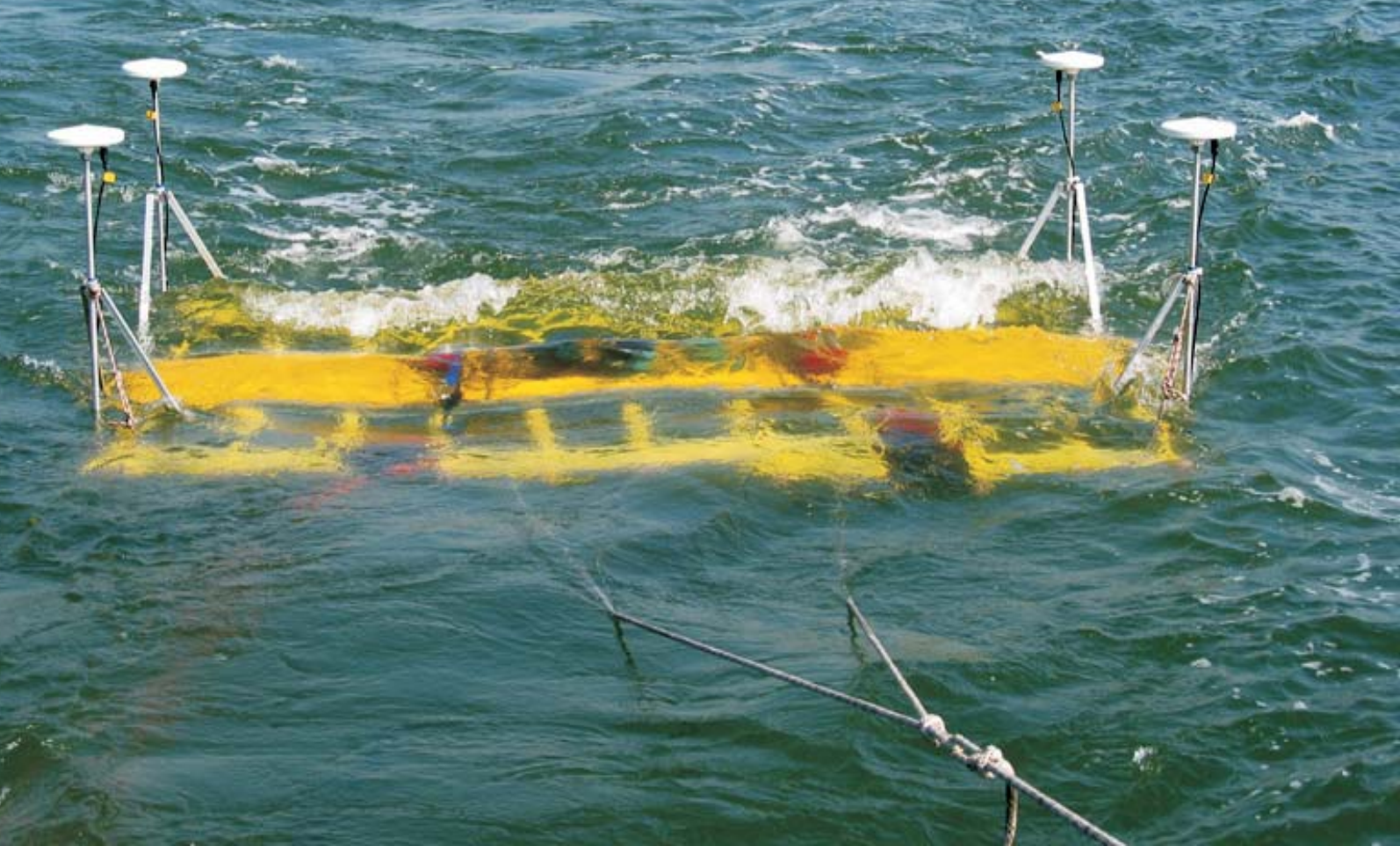

Bull et al. Figure 8 


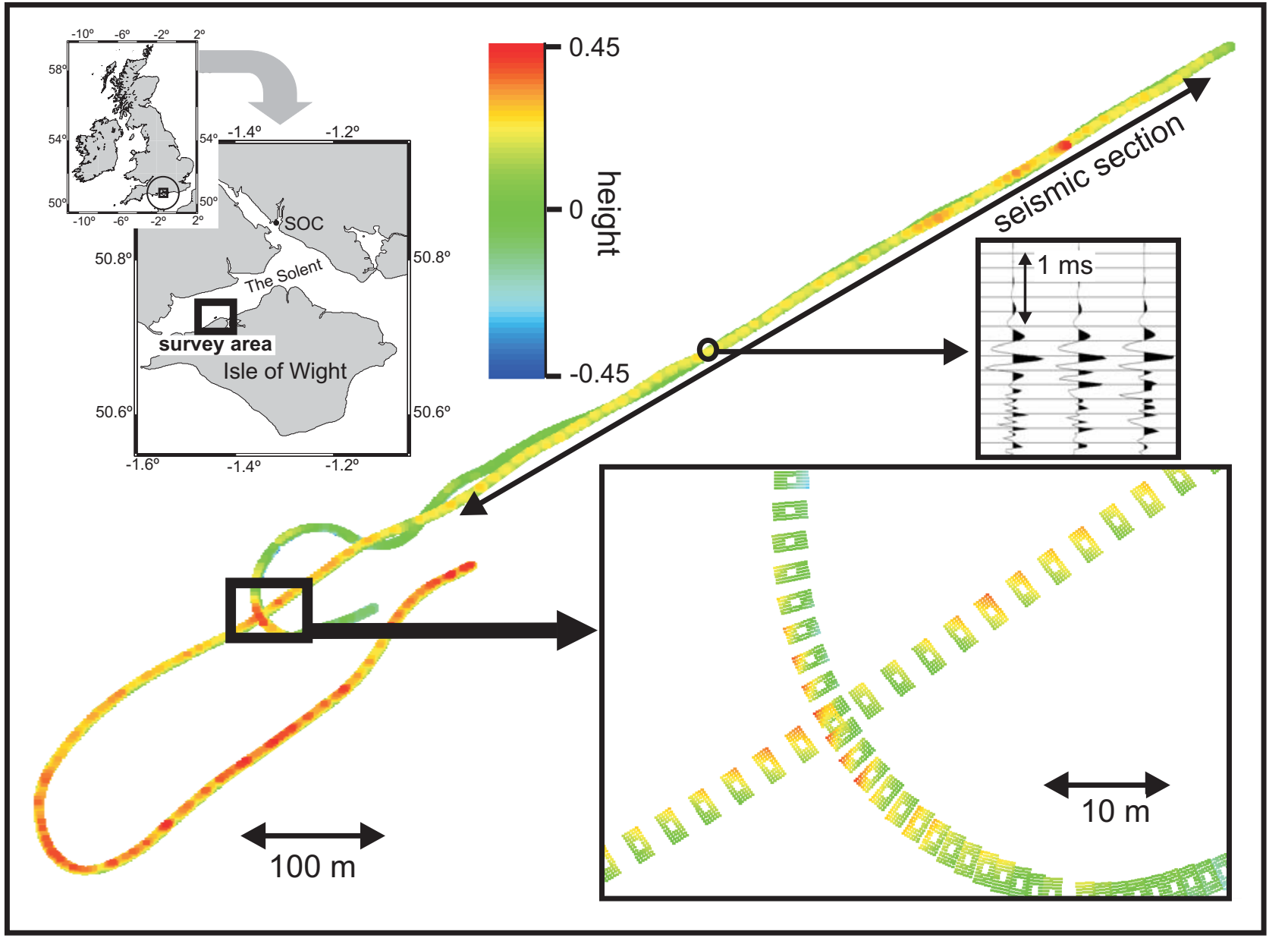

Bull et al. Figure 9 
